\title{
Improved maintenance for energy optimization in the calcium compound processing industry
}

\begin{abstract}
Malaysian industries, in general consider energy management a burden and non-profitable. Energy management initiatives are also viewed as a Cost-Centre. An Excel spreadsheet was therefore tailor-designed, which meets the requirements of the plant for energy optimization and management. The spreadsheet is grouped to reflect four parts, namely: plan, do, check and commit. This Plan-Do-Check-Commit cycle is based on the plan-do-check-act (PDCA) cycle. This spreadsheet has been developed with the objective of developing a tool that would be comprehensive, simple and flexible, open to be modified and tailored for the industries. The results indicated that there are opportunities for a more effective and structured approach to energy management in industry. An initial reduction of $5 \%$ in energy consumption has been recorded and it is the companyô responsibility to ensure that their energy management becomes part of their corporate culture.
\end{abstract}

Keyword: Energy consumption trends; Energy management system; Maintenance criteria 